\title{
Quantitative analysis of keratin filament networks in scanning electron microscopy images of cancer cells
}

\author{
M. BEIL, H. BR AXMEIER, * F. FLEISCHER, * V. SCHMIDT $\dagger \&$ \\ P. WALTHER $\ddagger$ \\ Department of Internal Medicine I, University Hospital Ulm, D-89070 Ulm, Germany \\ *Department of Applied Information Processing \& Department of Stochastics, \\ $\dagger$ Department of Stochastics, and $\doteqdot$ Electron Microscopy Facility, University Ulm, D-89069 Ulm, \\ Germany
}

Key words. Anisotropy, cytoskeleton, filaments, image analysis, keratin, scanning electron microscopy, skeletonization, statistical estimation.

\section{Summary}

The keratin filament network is an important part of the cytoskeleton. It is involved in the regulation of shape and viscoelasticity of epithelial cells. The morphology of keratin networks depends on post-translational modifications of keratin monomers. In-vitro studies indicated that network characteristics, such as filament crosslink density, determines the biophysical properties of the filament network. This report presents a quantitative method for the morphological analysis of keratin filament networks. Visualization of filaments was based on prefixation extraction of epithelial cells and scanning electron microscopy (SEM). SEM images were processed by a skeletonization algorithm to obtain a graph structure that represents individual filaments as well as their connections. This method was applied to investigate the effects of transforming growth factor $\alpha$ (TGF $\alpha$ ) on the morphology of keratin networks in pancreatic cancer cells. TGF $\alpha$ contributes to pancreatic cancer progression and activates signalling pathways phosphorylating keratin monomers. Using this new method, a significant alteration to the keratin network morphology could be detected in response to TGF $\alpha$.

\section{Introduction}

The filamentous cytoskeleton of eukaryotic cells consists of three polymer systems: microfilaments, microtubules and intermediate filaments. The intermediate filaments in epithelial cells are composed of keratins. Simple epithelia mainly express keratin 8 and 18 (Fuchs \& Weber, 1994). The major role of the intermediate filament cytoskeleton is the formation of a scaffold defining the shape and mechanical properties

Correspondence to: Professor Paul Walther. Tel.: +49 73150 23441; fax: +49 73150 23383; e-mail: paul.walther@medizin.uni-ulm.de of cells (Herrmann et al., 2003). However, the structure of this scaffold needs to be modulated dynamically to meet the changing needs of cells, e.g. during migration. The structure of keratin networks is regulated by post-translational modifications of keratin monomers, such as phosphorylation (Coulombe \& Omary, 2002; Strnad et al., 2002). These processes represent an important interface between biochemical signalling pathways and the keratin filament system. In-vitro studies with keratin suspensions have demonstrated that spatial arrangement patterns of filaments influence the mechanical properties of keratin networks (Coulombe \& Omary, 2002). In a previous study, we were able to establish a link between keratin phosphorylation, network architecture, viscoelastic properties and migration of cancer cells (Beil et al., 2003).

Morphological analysis of the filamentous cytoskeleton by light microscopy is based on the fact that the width of the filaments and the interfilament distances are smaller than the diffractionlimited resolution of optical microscopy. By combining electron microscopy with a prefixation extraction method, individual filaments can be visualized within cells, and the network architecture can thus be analysed at the level of its basic components (Ris, 1985; Svitkina \& Borisy, 1998). We have modified this method to image keratin filaments by scanning electron microscopy (SEM). Because keratin filaments are characterized by a high degree of biochemical stability (Zackroff \& Goldman, 1979), the specificity of this method for keratin filaments is ensured by high detergent concentrations during the extraction process. The next step was to develop a quantitative method to analyse the filament network architecture and its alterations in response to biochemical signals. In contrast to the approach chosen by Verkhovsky et al. (2003) restricting the analysis to the orientational order of filament networks, the image analysis method proposed in the present paper provides information about length and orientation of filaments as well as about the interconnections within the network. In particular, the method consists of a 
skeletonization of binarized images with a subsequent processing of the skeleton to remove artefacts. Finally, the skeleton structure is transformed into a graph to provide a quantitative analysis of the resulting line structure.

The new method was used to study the association between phosphorylation of keratin 8 and the keratin network architecture in pancreatic cancer cells. Keratin 8 is phosphorylated at Ser 431 by extracellular signal-regulated kinases (ERKs) (Omary et al., 1998). ERKs can be activated by incubating cells with transforming growth factor $\alpha$ (TGF $\alpha$ ), which plays a crucial role in pancreatic cancer progression (Seufferlein et al., 1999; Wagner et al., 2001).

\section{Materials and methods}

\subsection{Cell culture}

Panc1 human pancreatic cancer cells (American Type Culture Collection, Manassas, VA, U.S.A.) were grown on glass chamber slides. Cells were washed and subsequently incubated in serumfree medium for $1 \mathrm{~h}$ before being treated with $100 \mathrm{ng} \mathrm{mL}^{-1}$ TGF $\alpha$ (R\&D Systems, Minneapolis, MN, U.S.A.) for $30 \mathrm{~min}$.

\subsection{Electron microscopy}

The preparation of cells to visualize single keratin filaments by high-resolution SEM by a prefixation extraction method (Ris, 1985) was partially based on the protocol of Svitkina \& Borisy (1998). After washing the cells with phosphate-buffered saline (PBS), an extraction solution [1\% Triton X-100, 2.2\% PEG (molecular weight, $35 \mathrm{kDa}$ ), $50 \mathrm{~mm}$ imidazole, $50 \mathrm{~mm}$ potassium chloride, $0.5 \mathrm{~mm}$ magnesium chloride, 0.1 mm EDTA and 1 mu EGTA, pH 6.9] was added for $20 \mathrm{~min}$ at $4{ }^{\circ} \mathrm{C}$. Cells were rinsed four times with PBS and fixed with $4.0 \%$ formaldehyde (ultrapure EM grade, methanol free, Polysciences, Eppelheim, Germany) in 0.1 м cacodylate buffer ( $\mathrm{pH} 7.3$ ) for $10 \mathrm{~min}$ at room temperature. After rinsing with PBS, the slides were gradually dehydrated in propanol $(30 \%$, $50 \%, 70 \%, 90 \%$ and twice in $100 \%$ for $5 \mathrm{~min}$ at each step) and finally subjected to critical-point drying using carbon dioxide as transitional medium (Critical Point Dryer CPD 030, Bal-Tec,
Principality of Liechtenstein). Care was taken to prevent accidental air drying when changing the fluids. After drying, the cover slips were carefully cut to fit onto the Hitachi S-5200 specimen holders $(5 \times 8 \mathrm{~mm})$ using a home-made diamond cutter. The cut glass slides were then mounted on the Hitachi holders using double-sided tape and liquid silver paint to improve electrical conductivity. Samples were subsequently rotary coated with $3 \mathrm{~nm}$ of platinum-carbon by electron beam evaporation using a Bal-Tec Baf 300 freeze etching device (Bal-Tec) and imaged with a Hitachi S-5200 in-lens SEM (Tokyo, Japan) at an accelerating voltage of $4 \mathrm{kV}$ using the secondary electron signal. It was previously shown by immunoelectron microscopy that this method depletes actin filaments and microtubules and preserves keratin filaments (Beil et al., 2003).

To analyse the filamentous structure of the keratin network, sample regions were imaged at a primary magnification of $35000 \times$ (pixel size $2.63 \mathrm{~nm}$ ). These regions were chosen from the subcortical compartment of the cells directly adjacent to the cytoplasmic membrane. This procedure resulted in images containing a thin layer of filaments. In total we analysed 15 Panc1 cells (seven cells after incubation with TGF $\alpha$ and eight untreated cells). Figure 1 depicts typical Panc 1 cells from the TGF $\alpha$-treated group and untreated controls.

\subsection{Image segmentation}

As the skeletonization algorithm we used skeletonization by morphological thinning. The original structure is thinned by the use of a structuring element, e.g. a $3 \times 3$ matrix, that is rotated. This method is repeated until the structure remains unchanged for a whole cycle of rotated matrices. Homotopy, i.e. connectivity properties between different objects in the structure, is thereby preserved. For definitions of the basic morphological image operators, such as erosion, dilation, opening and closing, as well as algorithms for skeletonization of binary images see Jähne (2001), Serra (1988), Soille (2003) and references therein.

2.3.1. Classification of skeleton pixels. By counting the number of neighbouring pixels belonging to the skeleton it is possible to classify the pixels of the skeleton into endpoints (having

Fig. 1. SEMimages of Panc1 human pancreatic cancer cells after applying a prefixation extraction removing all non-keratin proteins. These overview images were acquired with a magnification of 3500×. (a) Untreated cells; (b) cells incubated with TGF $\alpha$.
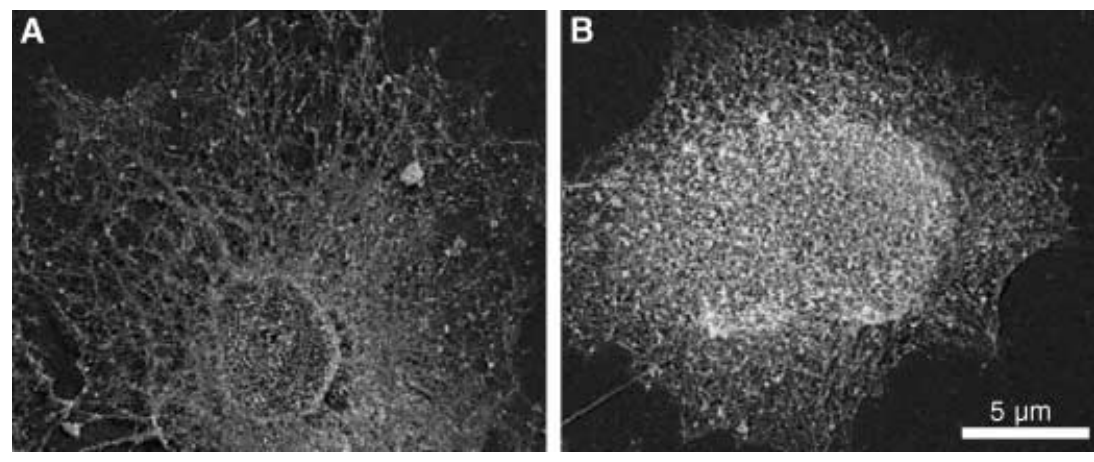


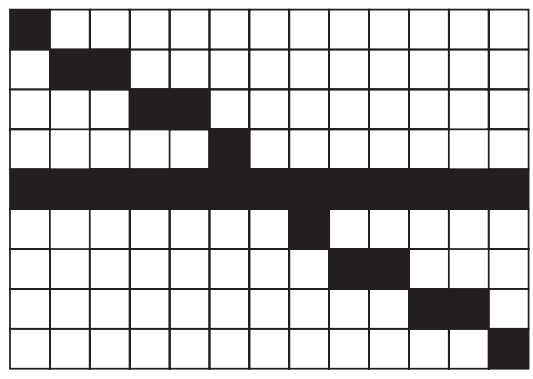

(a) Original skeleton

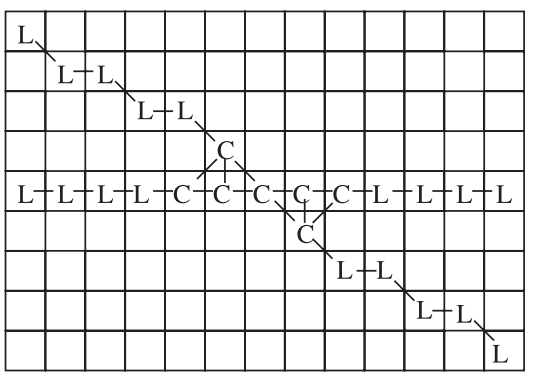

(b) Classification by number of neighbours

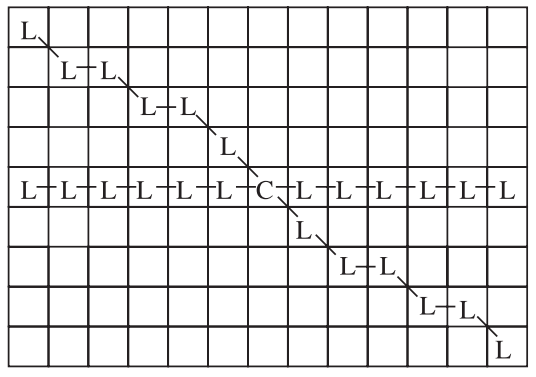

(c) Improved classification
Fig. 2. Classification and its modification, where C denotes crosspoints and L denotes linepoints. (a) Original skeleton; (b) classification by number of neighbours; (c) improved classification. only one neighbour), linepoints (having exactly two neighbours) and crosspoints (having more than two neighbours). In the following, using neigbour relationships, the connection between two crosspoints, a crosspoint and an endpoint or two endpoints is termed a connection path if otherwise only linepoints are involved. Note that often a classification using merely the number of neighbouring pixels might not be satisfactory because this would result in very many pixels classified as crosspoints and hence in very short connection paths between them. Therefore, certain criteria should be used in order to improve the results (see Fig. 2). In such an improved classification, homotopy should be preserved and the number of connection paths should be minimized. One criterion to decide between different possible solutions is to regard the difference in angle for the connections between one pixel and its neighbours, where angles close to $\pi$ are preferred. In contrast to methods which consider only the local neighbourhood of potential branching points (Beil et al., 1995), the approach considered in the present paper takes the topological properties of other line points into account.

2.3.2. Pruning. After reclassification, endpoints of the skeleton structure were detected. Because the planar filament network in our application is a network with closed branches, a pruning procedure is necessary to remove artefacts created by the preparation or imaging processes. A pruning procedure on the skeletons denotes the removal of all connection paths containing at least one endpoint and all the pixels of these connection paths. After pruning, pixels must be reclassified because crosspoints may have become linepoints.
2.3.3. Transformation of the skeletoninto a graph. Assuming that connections between crosspoints are linear, the pruned skeleton can be transformed into a graph or, seen from a different viewpoint, into a line segment structure, in which each pair of crosspoints that has a connection path is represented as a line segment between them (see Fig. 3a,b).

2.3.4. Modification of line segment structure. To remove artefacts resulting from loops (connection paths where starting point and endpoint are identical) or from two filaments crossing each other and enclosing a small angle (see Fig. 3), the line structure is modified by merging two or more crosspoints into a single one using the centre of gravity of the involved crosspoints as a new crosspoint. This step is performed for crosspoints a distance of less than $d_{\max }$ apart and in an ascending sequence, meaning that crosspoints a smaller distance apart are merged first.

2.3.5. Algorithm description. Figure 4 depicts intermediate results of the algorithms implemented. The greyscale images are transformed into binary images by using a threshold $t=\bar{f}$, where $\bar{f}$ represents the mean greyscale value within the image. On these binary images an opening is performed in order to reduce noise effects on the filament topology of the image objects. The binary images are then skeletonized. The resulting skeletons are pruned and transformed into line segment structures. Finally, these line segment structures are modified by merging nearby crosspoints ( $d_{\max }=8$ pixels) to obtain line segment structures that can be statistically analysed. 
Fig. 3. Transformation and improvement of the structure. (a) Typical skeleton of two filaments crossing each other at a small angle; (b) transformation into graph structure; (c) merging of nearby crossings.

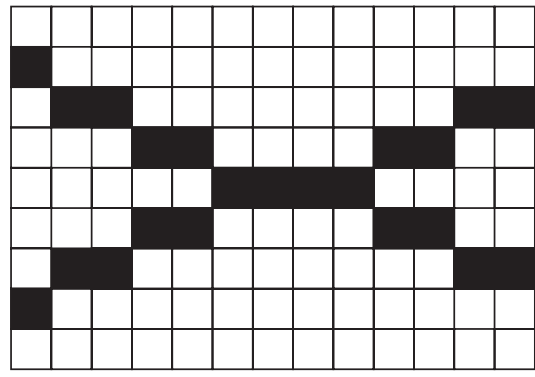

(a) Typical skeleton of two filaments crossing each other at a small angle

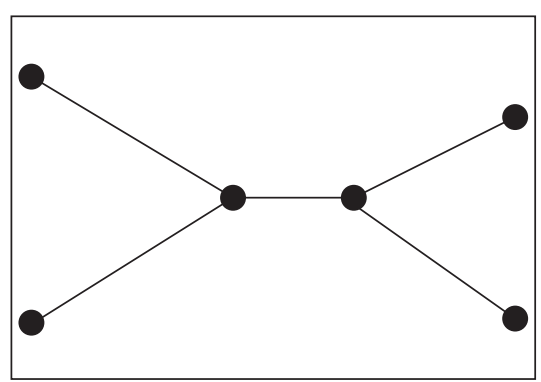

(b) Transformation into graph structure

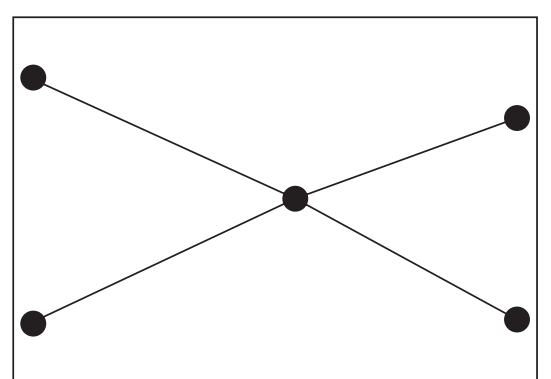

(c) Merging of nearby crossings

\subsection{Statistical analysis}

The line segments produced by the algorithm described in Section 2.3 were analysed statistically with respect to characteristics such as the number of line segments, their length and orientation distributions. With regard to orientation distributions, the analysis was performed on circular cutouts with radii of 300 pixels $(\approx 0.79$ $\mu \mathrm{m})$ centred at the midpoints of the images. A circular sampling window was chosen to avoid any bias with respect to orientation distributions. For the analysis of segment lengths, sampling windows were chosen to be quadratic with a side length of 500 pixels $(\approx 1.32 \mu \mathrm{m})$ centred at the midpoints of the images. Orientation distributions were tested for uniformity by using Kuiper's test (Mardia \& Jupp, 2000), while statistical comparison of groups was based on the Wilcoxon-Mann-Whitney test.

2.4.1. Estimation of segment lengths andorientationangle. Segment lengths were estimated by using the Euclidean distance between the two segment endpoints. Segment lengths were taken into account if the lexicographically smaller endpoint of the segment was located inside the sampling window. The sampling window was chosen to be sufficiently small in order to avoid edge effects caused by an inability to observe whole lengths of relevant segments. Orientations of segments were determined as angles between segments and the $x$-axis, where only segments with their lexicographically smaller endpoint lying in the sampling window were considered.

2.4.2. Orientation analysis. The main aim of the orientation analysis was to determine the degree of anisotropy, i.e. the degree of non-uniformity, of the orientation distributions of the detected line segments for the two different groups. In order to analyse the orientation distributions, two different approaches were considered. As a first approach, the circular standard deviation was computed, whereas in the second approach an index of dispersion was estimated by using resampling techniques. Note that for both approaches we considered the typical point method, meaning that each line segment is weighted with the same weight, independent of its length.

2.4.3. Circular variance and circular standard deviation. For a more detailed explanation of the characteristics described here, see, for example Batchelet (1981) and Mardia \& Jupp (2000). Let $\theta_{1}, \ldots, \theta_{n}$ denote a sample of line segment angles with respect to the $x$-axis and with corresponding unit vectors $u_{1}, \ldots, u_{n}$. Then the mean direction $\bar{\theta}$ of $\theta_{1}, \ldots, \theta_{n}$ is the direction of the resultant $u_{1}+\ldots+u_{n}$ of $u_{1}, \ldots, u_{n}$. It is also the direction of the centre of mass $\bar{u}$ of $u_{1}, \ldots, u_{n}$, where $\bar{u}$ can be described in Cartesian coordinates as

$$
\bar{u}=(\bar{C}, \bar{S})
$$

with

$$
\bar{C}=\frac{1}{n} \sum_{j=1}^{n} \cos \theta_{j}
$$

and

$$
\bar{S}=\frac{1}{n} \sum_{j=1}^{n} \sin \theta_{j}
$$




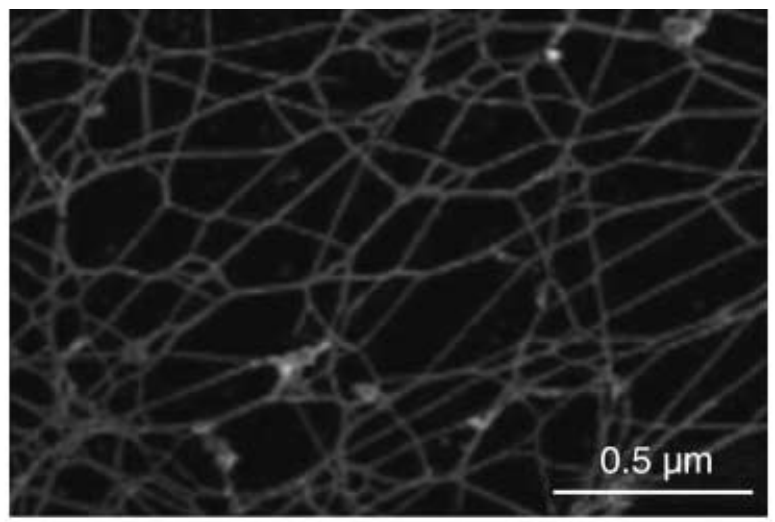

(a)

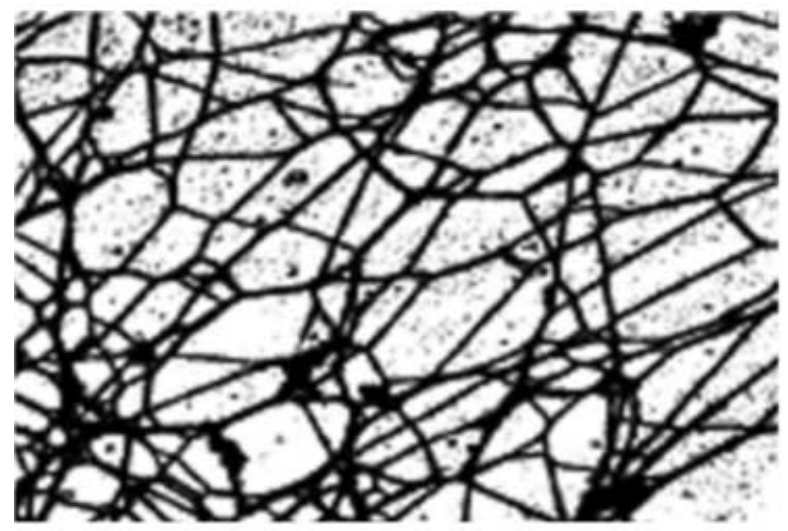

(b)

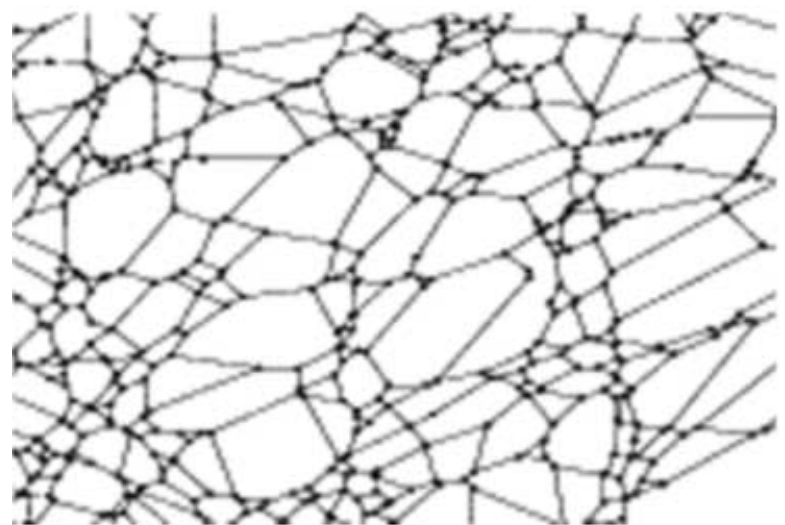

(d)

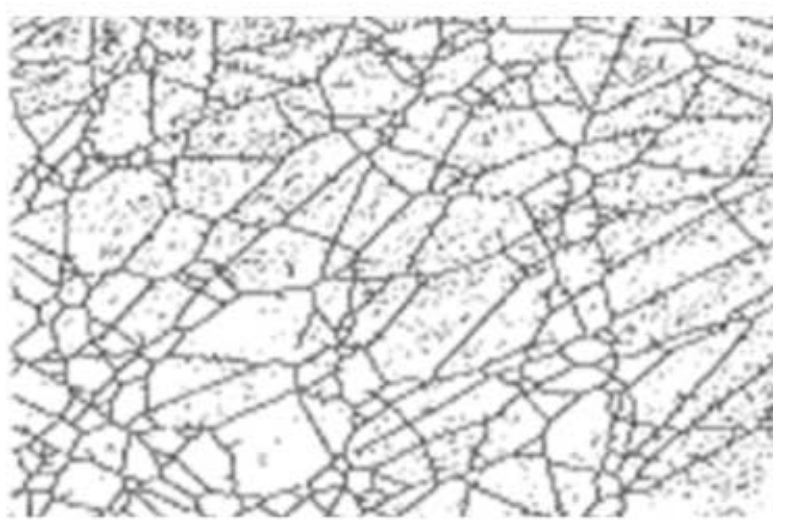

(c)

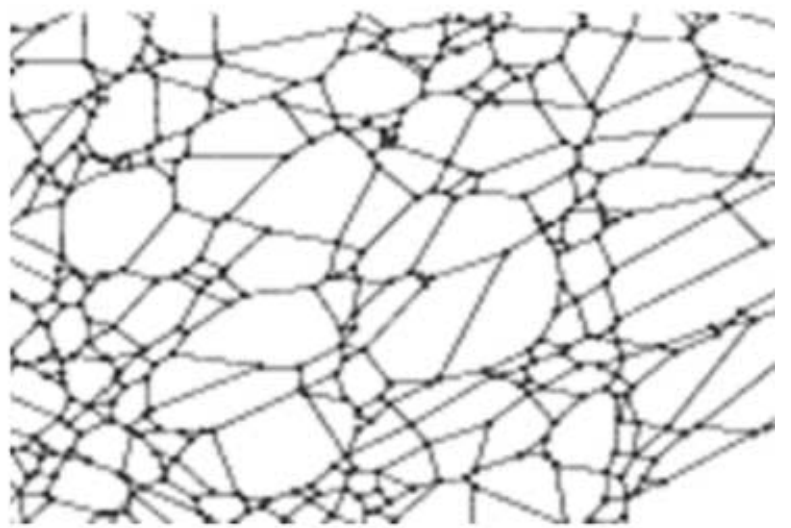

(e)

Fig. 4. Intermediate steps in the image segmentation. (a) Greyscale sample image; (b) binary image after thresholding; (c) skeleton of binary image; (d) graph structure after pruning; (e) graph structure after merging nearby crossings. 
Hence, $\bar{\theta}$ is the solution of the system of equations

$$
\bar{C}=\bar{R} \cos \bar{\theta}, \quad \bar{S}=\bar{R} \sin \bar{\theta}
$$

(provided that $\bar{R}>0$ ), where the mean resultant length $\bar{R}$ is given as

$$
\bar{R}=\left(\bar{C}^{2}+\bar{S}^{2}\right)^{1 / 2} .
$$

The sample circular variance is defined as

$$
V=1-\bar{R}
$$

and can be used as a measure of dispersion because if the noberved directions are tightly clustered about the mean direction $\bar{\theta}$, then the sample circular variance will be nearly zero. On the other hand, if the directions are widely dispersed then $V$ will be almost 1 .

An analogue for circular data of the standard deviation on the line is given by the sample circular standard deviation, defined by

$$
\sigma=(-2 \log (1-V))^{1 / 2}
$$

and which has similar dispersion measure properties as $V$. Therefore, if $\theta_{1}=\left(\theta_{11}, \ldots, \theta_{1 n_{1}}\right), \ldots, \theta_{m}=\left(\theta_{m 1}, \ldots, \theta_{m n_{m}}\right)$ denote $m$ samples of line segment angles with sizes $n_{1}, \ldots, n_{m}$, respectively, we compute the sample circular standard deviations $\sigma_{1}, \ldots$, $\sigma_{m}$ in order to obtain information regarding the orientation distributions.

2.4.4. Index of dispersion. As a confirmation for the results obtained for the sample circular standard deviation, we regarded a further parameter for the degree of anisotropy of the line segments constructed as follows. Let

$$
n_{\min }=\min _{i=1, \ldots, m} n_{i}
$$

denote the minimal size of the $m$ samples of angles $\theta_{1}=\left(\theta_{11}, \ldots\right.$, $\left.\theta_{1 n_{1}}\right), \ldots, \theta_{m}=\left(\theta_{m 1}, \ldots, \theta_{m n_{m}}\right)$. Now for each sample $\theta_{1}$ a subsample of $n^{*}$ angle values is drawn without repetition, where $n^{*} \leq n_{\min }$. This procedure is repeated $l$ times. For each of these $l$ samples of size $n^{*}$ the index of dispersion $D_{i, j}$ is given by

$$
D_{i, j}=\frac{1}{S} \sum_{k=1, \ldots, s}\left(x_{k}-n^{*} p_{k}\right)^{2}, \quad i=1, \ldots, m, \quad j=1, \ldots, l,
$$

where $s$ is the number of classes, i.e. the number of directional sectors considered, $x_{k}$ is the number of angles of the subsample belonging to the $k$ th class, and $p_{k}$ is the probability of belonging to the $k$ th class under the assumption of isotropy. The mean index of dispersion $D_{i}$ is then given by

$$
D_{i}=\frac{1}{l} \sum_{j=1, \ldots, l} D_{i, j}
$$

These mean indices of dispersion can be regarded as anisotropy parameters reflecting the test statistic for the chi-squared goodness-of-fit test where by a randomized drawing procedure it is ensured that samples are of equal size. Note that in contrast to the circular standard deviation, a small value of $D_{i}$ indicates that angles are almost uniformly distributed. Hence if $D_{i}$ is small it can be assumed that the angles have a completely random distribution, whereas large values of $D_{i}$ indicate that there are preferred directions with respect to the orientation of the line segments. For later analysis we used $s=16$ classes and $l=1000$ iterations.

2.4.5. Software. All software developed and used for image analysis as well as for statistical analysis was included in the GeoStoch library system. GeoStoch is a Java-based openlibrary system developed by the Department of Applied Information Processing and the Department of Stochastics of the University of Ulm, and which can be used for stochasticgeometric modelling and spatial statistical analysis of image data (Mayer, 2003; Mayer et al., 2004; http://www.geostoch. de).

\section{Results}

\subsection{Visual results}

Figure 5 shows the results of the segmentation algorithm for three different sample images that have an ascending degree of structural complexity. Problems occurred in cases where spaces between filaments were extremely narrow, i.e. filaments were touching each other, or if filament connections could not be further differentiated even by a human observer. These examples also indicated that the assumption of piecewise linearity of connections between crosspoints might be a reasonable choice because most of these connections were quite small and longer connections tended to be approximately linear. Moreover, most but not all branching points represented interconnections of keratin filaments. An inspection of the segmentation results revealed that $9.8 \%$ of all branching points $(10.3 \%$ in the control group, $9.5 \%$ in the TGF $\alpha$-treated group) resulted from cross-over of long filaments located in different planes.

\subsection{Number of segments and segment lengths}

Figure 6 shows for both groups (untreated controls, cells treated with TGF $\alpha$ ) a sample histogram of segment lengths for a specific image, as well as a cumulated histogram for all segment lengths with respect to all images of a group. Although the shape of the length distribution seems to be quite similar, the histograms indicate that mean segment lengths are reduced in the case of the TGF $\alpha$-treated group compared with the control group. Note that due to the applied algorithm the minimal length of a detected segment equals $d_{\max }=21 \mathrm{~nm}$ and that the mean 


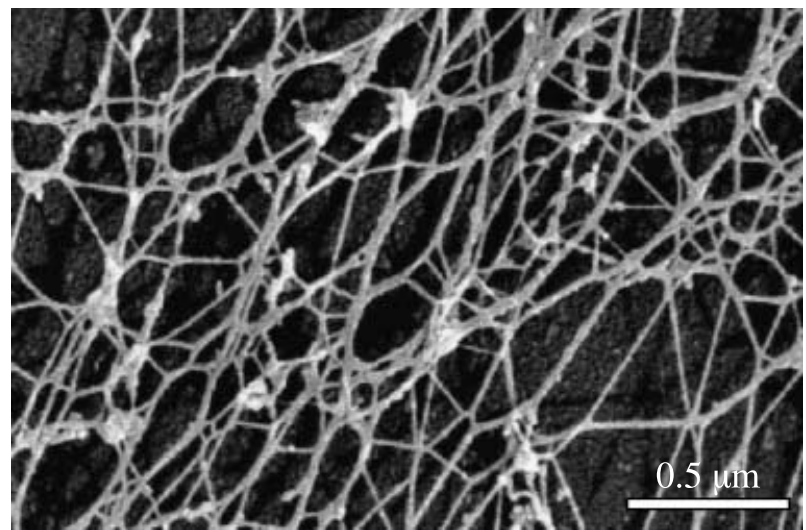

Original image from control group

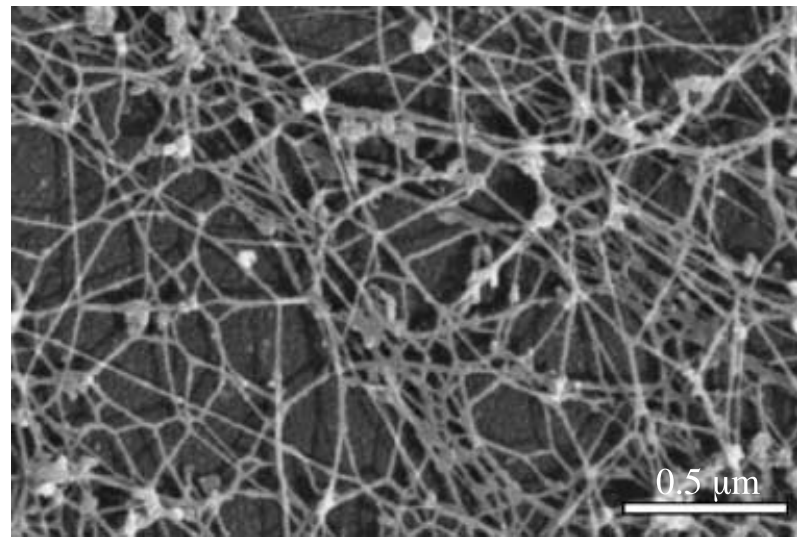

Original image from TGF $\alpha$ group

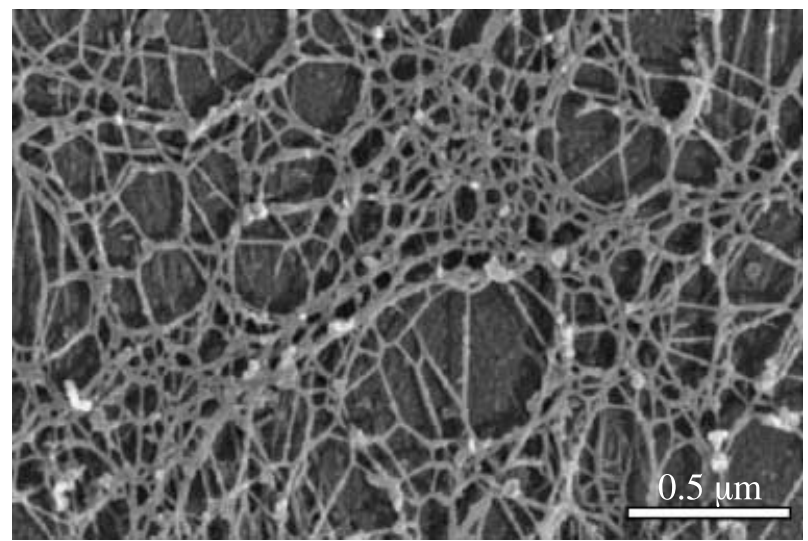

Original image from TGF $\alpha$ group

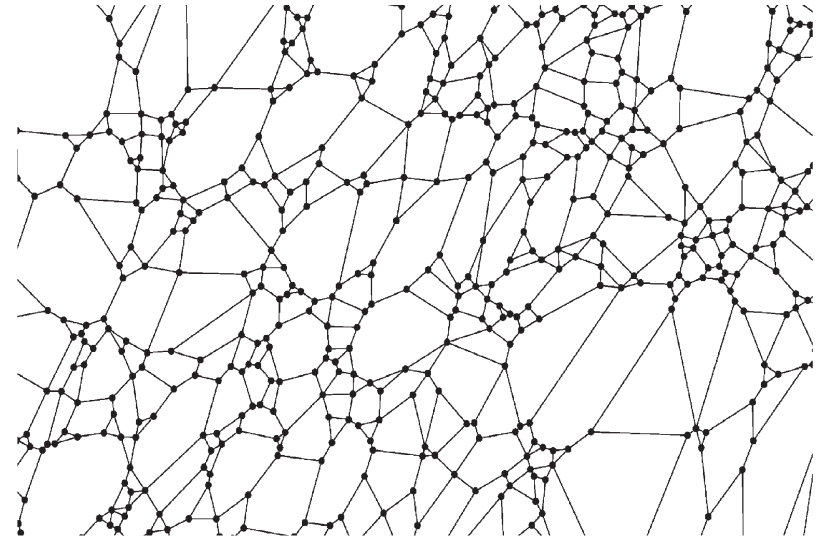

Graph structure

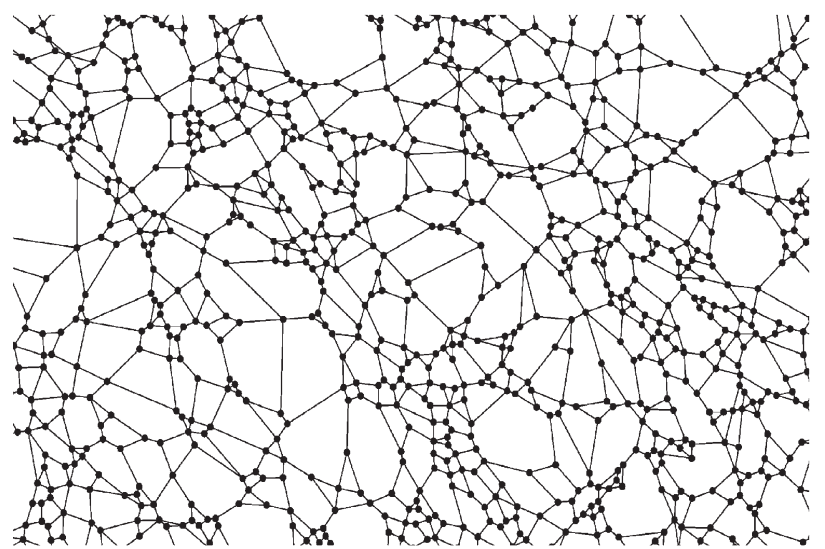

Graph structure

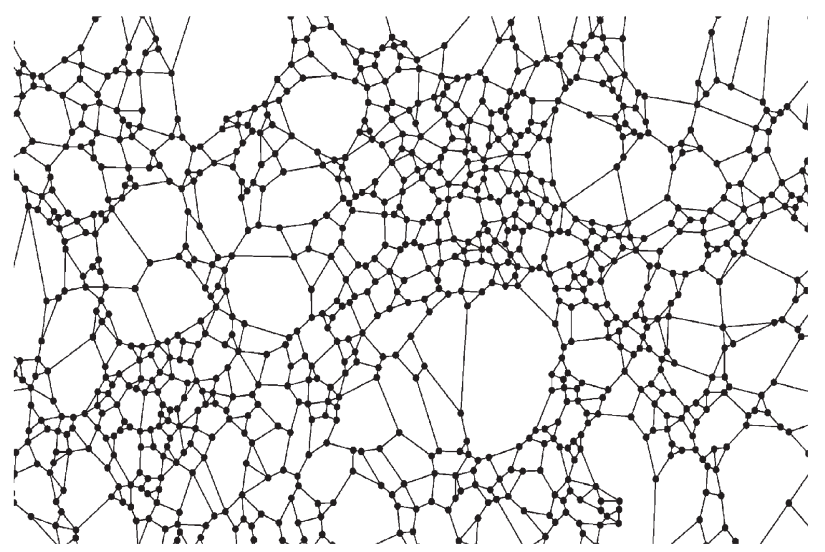

Graph structure

Fig. 5. Results of the segmentation algorithm.

number of detected segments is thereby reduced. In Fig. 7 boxplots for the total number of segments and the mean segment length per sampling region are displayed for both the TGF $\alpha$ incubated group of cells and the control group. Statistical tests for the two groups on the equality of the mean number of segments and on the equality of mean segment length, respectively, were performed and both were rejected at a test level of $\gamma=0.05$. Note that there is no principle difference in regarding 

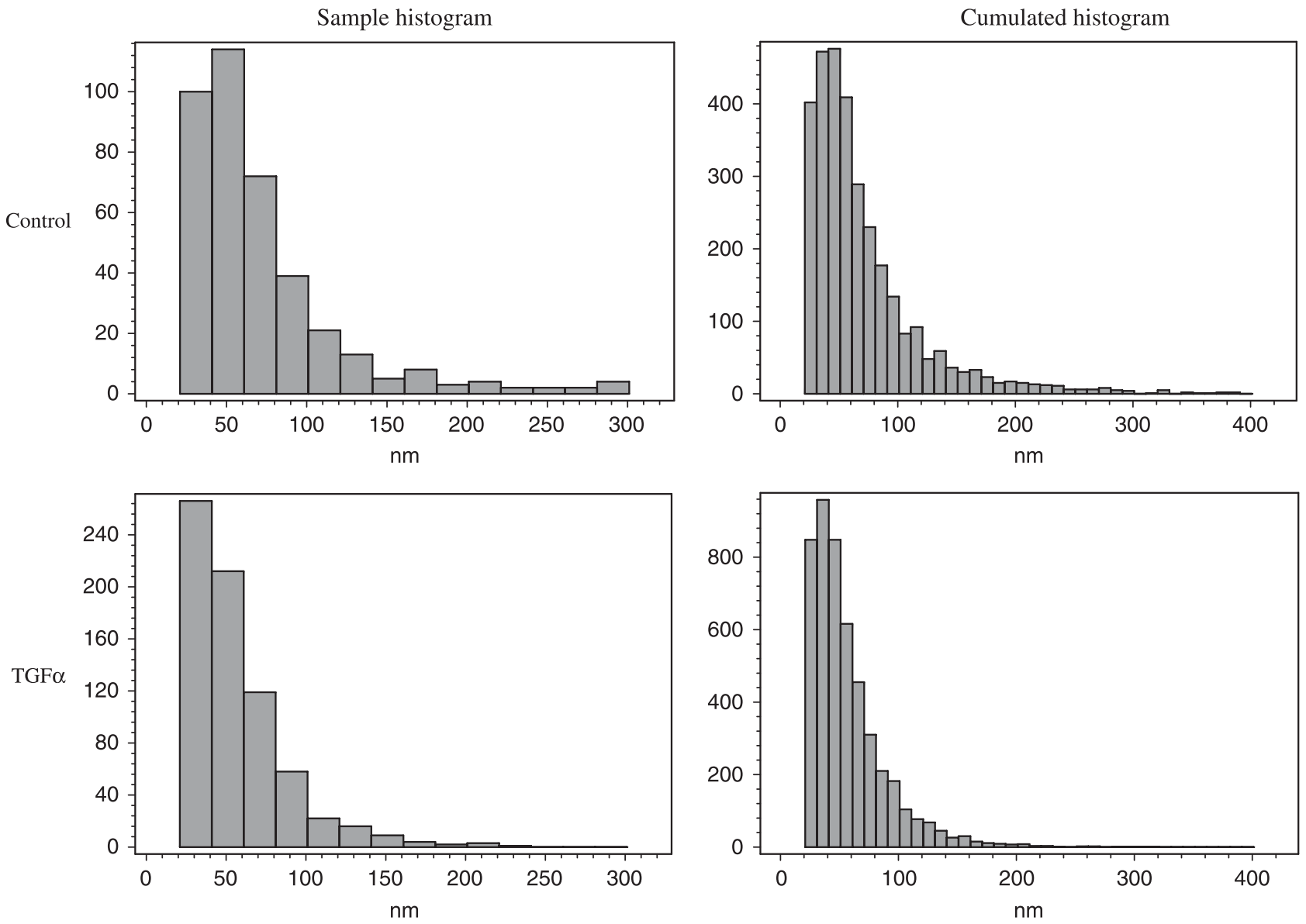

Fig. 6. Histograms of segment length.

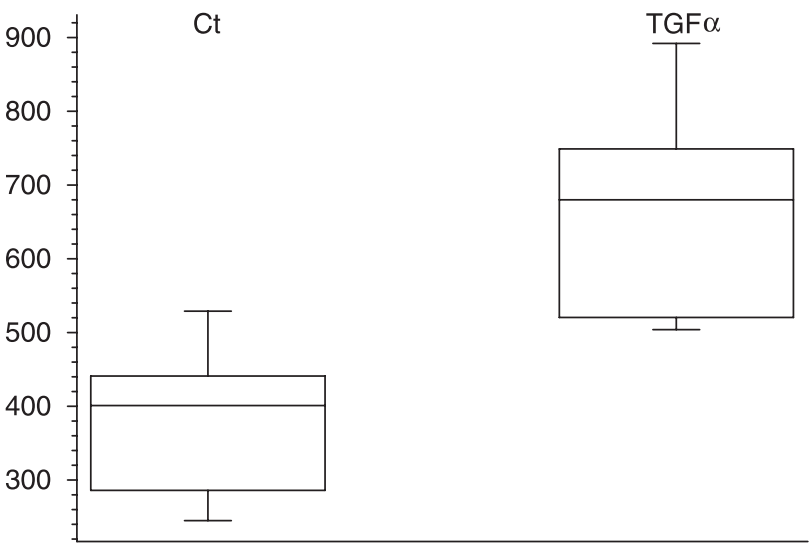

(a) Mean number of segments

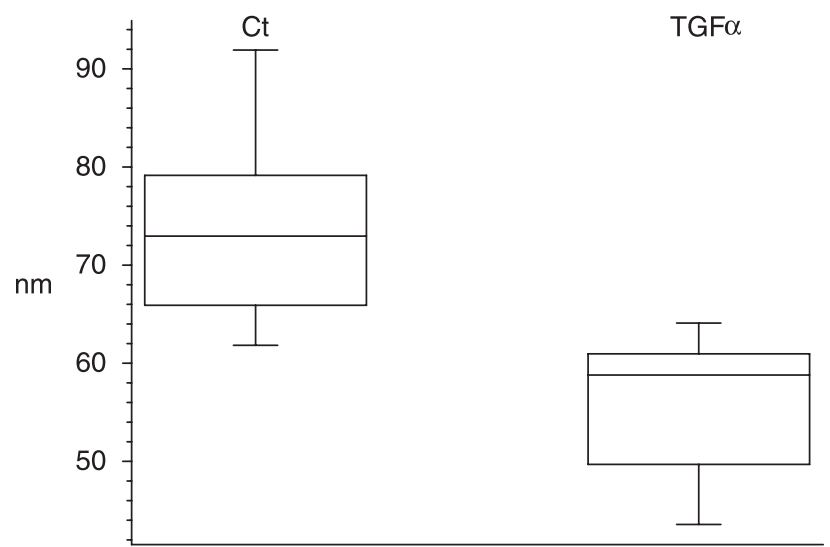

(b) Mean segment length

Fig. 7. Boxplots of the total number of segments and mean segment length (the median is depicted as the line in the middle of the box, the first and third quartiles as the upper and lower border of the box, and the minimum and maximum value as the upper and lower hook).

total mean numbers instead of densities, i.e. mean numbers per unit area, because the sampling windows were of a fixed size. Furthermore, note that the filament number and density are correlated strongly with crosslink density.

\section{3. Orientation distributions and anisotropy}

The orientation of a line segment can be described by its angle $\theta$ to the $x$-axis with $\theta \in[-\pi / 2, \pi / 2]$. Note that we have axial data 

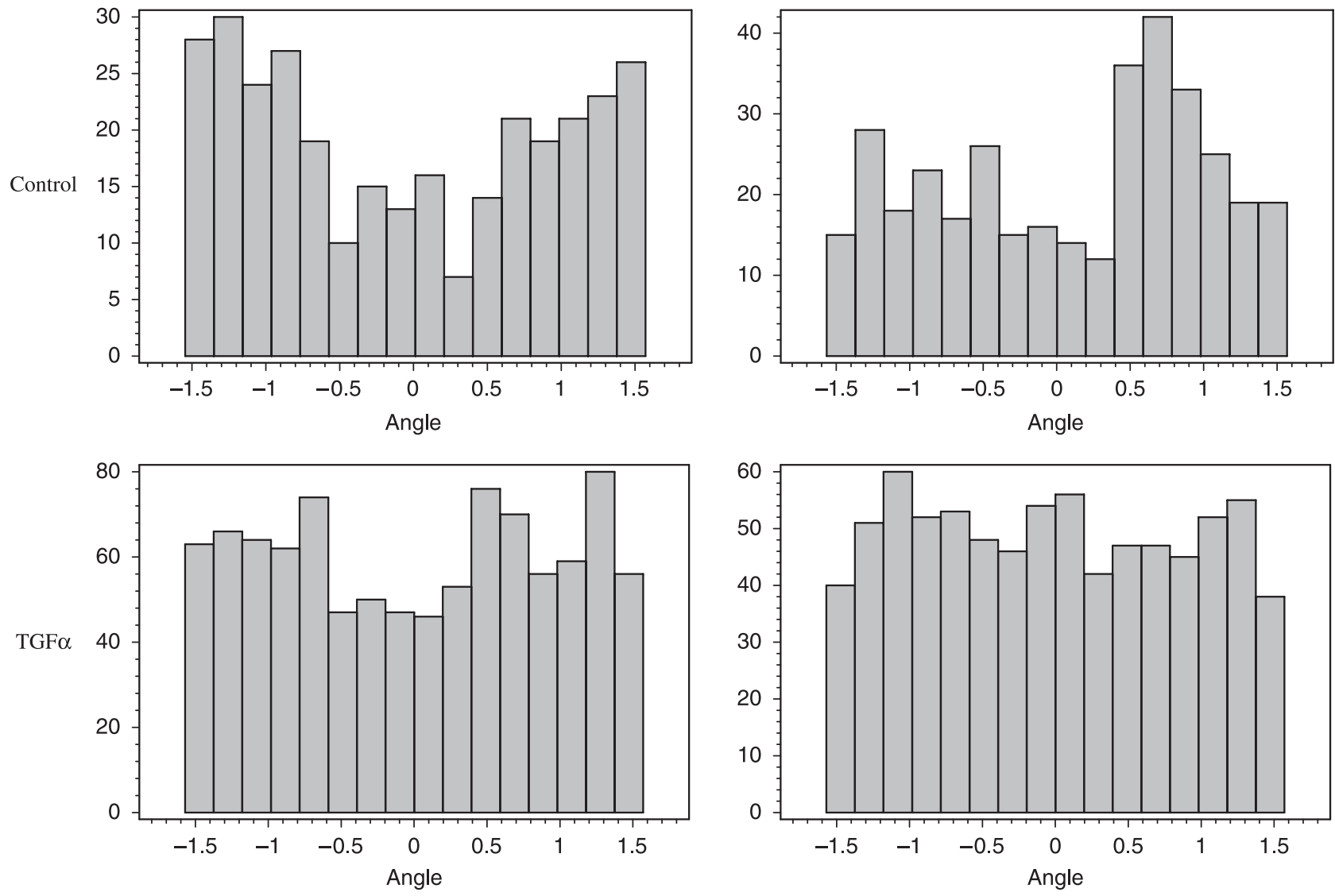

Fig. 8. Histograms of segment orientations as depicted by its angle with the $x$-axis.

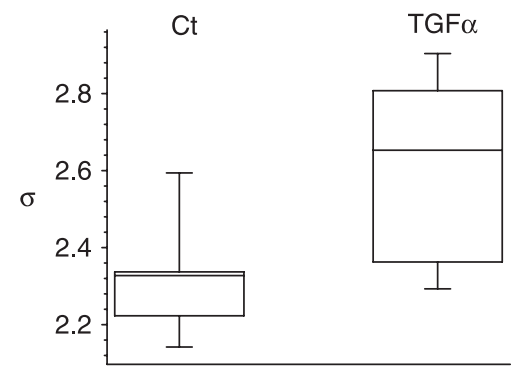

(a) Circular standard deviation

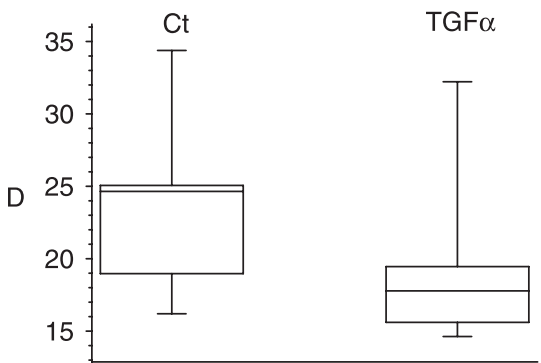

(b) Index of dispersion
Fig. 9. Boxplots of circular standard deviation and boxplots of indices of dispersion (the median is depicted as the line in the middle of the box, the first and third quartiles as the upper and lower border of the box, and the minimum and maximum value as the upper and lower hook). given, meaning that a specific line segment has two possible directions. Figure 8 displays sample histograms for the two groups. Although there is a strong variability within each group, the orientation distribution in the case of the TGF $\alpha$ incubated cells tends to be more uniform than in the untreated cells. Statistical tests for uniform orientation distribution show that for the control group $7 / 8$ sample images lead to a rejection of the hypothesis of uniform orientation distribution, whereas for the TGF $\alpha$-incubated cells only $4 / 7$ sample images caused a rejection $(\gamma=0.05)$. In Fig. 9(a,b) boxplots of the circular standard deviation described in Eq. (2.7) as well as boxplots of the index of dispersion described in Eq. (2.9) as anisotropy parameters are displayed for the two groups. Statistical tests for equality of expected circular standard deviations and for equality of expected indices of dispersion lead in both cases to rejection $(\gamma=0.05)$, meaning that the expected circular standard deviation is greater for the TGF $\alpha$-treated group than for the control group. This result is confirmed by the fact that the opposite is true for the expected indices of dispersion. Hence, filaments of the control group seem to be more uniformly orientated than those of the TGF $\alpha$-treated group. 


\section{Discussion}

The cytoskeleton is involved in essentially all structural and dynamic aspects of living cells (Ramaekers \& Bosman, 2004). Morphological features of the intermediate filament cytoskeleton were shown to determine the biomechanical properties of filament networks, which control cell shape and motility as well as tissue stability (Goldman et al., 1996; Bruel et al., 2001; Ma et al., 2001; Coulombe \& Omary, 2002). We have shown in a previous report that extensive remodelling of the keratin network in response to sphingosylphosphorylcholine changes the viscoelastic modulus, i.e. the force required to stretch a cell a set distance, of human pancreatic cancer cells, overriding even the impact of microfilament reorganization and possibly resulting in an enhanced metastatic activity of pancreatic cancer cells (Beil et al., 2003). TGF $\alpha$ was shown to be also involved in pancreatic cancer progression (Wagner et al., 2001). In the present study, we therefore investigated the role of TGF $\alpha$ in the structure of the keratin filament network by using a keratinspecific prefixation extraction method and SEM.

We observed distinct changes of the keratin network architecture in Panc1 cells in response to TGF $\alpha$. The focus of our investigation was then to quantify the structural alterations of the keratin network at the cell periphery. This region appears to be an important centre of spatially defined keratin organization given that de novo filament formation was observed in this compartment (Windoffer et al., 2004). Thus, stimulated changes of the network architecture might become visible first in this cytoplasmic region. Furthermore, the subcortical compartment represents an interface connecting the cytoskeleton and intracellular signalling pathways with transmembrane receptors mediating contact to other cells and the extracellular matrix. Thus, this region plays an important role in cell-cell interactions and cell migration (Wehrle-Haller \& Imhof, 2003). A better understanding of the modulation of these aspects of cancer cell biology by TGF $\alpha$ could thus reveal new therapeutic targets to prevent progression of the disease. Because the structural changes after a short incubation with TGF $\alpha$ were expected to be subtle, the morphological investigation required a sensitive method for the analysis of keratin filaments and the characteristics of their spatial distribution.

The biochemical stability of keratin filaments permitted the application of high detergent concentrations during the extraction phase washing out almost all non-keratin molecules from the cytoplasm. We cannot exclude that this approach also removed some of the keratin filaments with a decreased stability during filament turnover. However, experiments with lower detergent concentrations resulted in a very similar filament density but with a large number of protein precipitates composed of other abundant cellular proteins such as actin. After applying critical-point drying and platinum coating, SEM produced highcontrast images of the keratin cytoskeleton. The availability of whole cells provided the opportunity to select specifically subcortical regions for further analysis.
The image analysis method implemented in this study was based on a skeletonization of binarized images with a subsequent pruning of the line structure and reconstruction of branching points. Owing to the sufficiently high contrast of SEM images, the segmentation algorithm yielded a good quality match between the resulting line structure and the filament network in the original greyscale images. In images with a lower signalto-noise ratio, methods of greyscale skeletonization might be useful (Meyer, 1989; Vincent \& Soille, 1991). The morphological analysis of the line structure, i.e. filament network, involved filament number, length distribution and spatial orientation of filaments. During slide processing, partial collapse of the keratin network can occur, inducing displacement of the filaments. In this setting, statistical features of filament density and network connectivity are considered to be more stable than features describing the localization of individual filaments. In experiments in which parallel filaments tend to aggregate, the segmentation method applied for filament detection is prone to underestimate the density of filaments. This could introduce a bias into the statistical analysis depending on network morphology. Misclassification of filament crossovers as branching points represented another potential problem. However, in our experimental setting, misclassifcation rates were similar between controls and stimulated cells and, thus, did not invalidate the comparison between these two groups.

Using statistical features of network morphology we found a significant difference in the subcortical keratin network architecture between Panc1 exposed to TGF $\alpha$ and controls. Such a remodelling of the peripheral keratin network could contribute to an enhanced cell migration induced by TGF $\alpha$, as was recently described for ovarian cancer (Sewell et al., 2005). Note that, in addition to the morphological analysis performed in the present paper, an investigation of the detected branching points with respect to their spatial distribution might be of interest. In this context, methods similar to those used in Beil et al. (2005) can be applied. Point process characteristics, such as the pair correlation function or the L-function, both with respect to the point pattern generated by the branching points, might be analysed. Such an approach may provide information about attraction or repulsion effects between pairs of branching points within specific distance regions. For an introduction into this type of analysis, see, for example, Stoyan et al. (1995).

Several studies have already investigated the intermediate filament cytoskeleton using light microscopy and quantitative image analysis. In a previous report, density and orientation of detectable filaments were found to change during development of the fetal rat liver (Vassy et al., 1996). The perinuclear organization of keratins in cancer cells appears to be regulated by gravity (Vassy et al., 2001). In a study by Helmke et al. (2003), a map of the distribution of intracellular strain was computed from the displacement of intermediate filaments in endothelial cells exposed to shear stress. However, the analysis of light microscopy images is confined to thick bundles of 
filaments and, thus, excludes potentially relevant components of the cytoskeleton. This restriction also affects the comparison of real image data with the results from experiments simulating the synthesis of filament networks (Portet et al., 2003). Investigations into model systems of interconnected filaments have identified the crosslink density as an important parameter determining the biophysical properties of networks (Head et al., 2003). In contrast to light microscopy studies, our method provides the opportunity to image individual filaments as well as their crosslinks. By using this approach, data obtained from real cells can be compared with the results from simulation experiments to evaluate the role of structural features in the biophysical characteristics of cytoskeletal networks. However, SEM-based imaging studies are restricted to experiments in which structural changes do not need to be monitored in time for individual cells. Furthermore, the analysis of network morphology can be combined with biochemical data on post-translational modifications of keratin monomers to gain insights into the regulation of the keratin cytoskeleton by biochemical signalling pathways.

In conclusion, we have developed an image analysis method to investigate the morphology of the keratin cytoskeleton in cultured cells. The visualization of individual filaments was based on a prefixation extraction and SEM. Using this new method, distinct changes of the keratin network in pancreatic cancer cells could be detected in response to TGF $\alpha$.

\section{Acknowledgements}

We thank Elke Wolff-Hieber and Eberhard Schmid for expert technical assistance. H.B. is supported by a grant of the Graduiertenkolleg 460 at the University of Ulm. This project was partly supported by the Deutsche Forschungsgemeinschaft (SFB 518).

\section{References}

Batchelet, E. (1981) Circular Statistics in Biology. Academic Press, London. Beil, M., Fleischer, F., Paschke, S. \& Schmidt, V. (2005) Statistical analysis of $3 \mathrm{D}$ centromeric heterochromatin structure in interphase nuclei. $J$. Microsc. 217, 60-68.

Beil, M., Irinopoulou, T., Vassy, J. \& Wolf, G. (1995) A dual approach to structural texture analysis in microscopic cell images. Comput. Meth. Programs Biomed. 48, 211-219.

Beil, M., Micoulet, A., von Wichert, G., Paschke, S., Walther, P., Omary, M.B., Van Veldhoven, P.P., Gern, U., Wolff-Hieber, E., Eggermann, J., Waltenberger, J., Adler, G., Spatz, J. \& Seufferlein, T. (2003) Sphingosylphosphorylcholine regulates keratin network architecture and viscoelastic properties of human cancer cells. Nat. Cell Biol. 5, 803-811.

Bruel, A., Paschke, S., Jainta, S., Zhang, Y., Vassy, J., Rigaut, J.P. \& Beil, M. (2001) Remodeling of vimentin cytoskeleton correlates with enhanced motility of promyelocytic leukemia cells during differentiation induced by retinoic acid. Anticancer Res. 21, 3973-3980.

Coulombe, P.A.\& Omary, M.B. (2002) 'Hard' and 'soft' principles defining the structure, function and regulation of keratin intermediate filaments. Curr. Opin. Cell Biol. 14, 110-122.
Fuchs, E. \& Weber, K. (1994) Intermediate filaments: structure, dynamics, function and disease. Annu. Rev. Biochem. 63, 345-382.

Goldman, R.D., Khuon, S., Chou, Y.H., Opal, P. \& Steinert, P.M. (1996) The function of intermediate filaments in cell shape and cytoskeletal integrity. J. Cell. Biol. 134, 971-983.

Head, D.A., Levine, A.J. \& MacKintosh, F.C. (2003) Deformation of crosslinked semiflexible polymer networks. Phys. Rev. Lett. 91, 108102, 1-4.

Helmke, B.P., Rosen, A.B. \& Davies, P.F. (2003) Mapping mechanical strain of an endogenous cytoskeletal network in living endothelial cells. Biophys. J. 84, 2691-2699.

Herrmann, H., Hesse, M., Reichenzeller, M., Aebi, U. \& Magin, T.M. (2003) Functional complexity of intermediate filament cytoskeletons: from structure to assembly to gene ablation. Int. Rev. Cytol. 223, 83175.

Jähne, B. (2001) Digital Image Processing. Springer, Berlin.

Ma, L., Yamada, S., Wirtz, D. \& Coulombe, P.A. (2001) A 'hot-spot' mutation alters the mechanical properties of keratin filament networks. Nat. Cell Biol. 3, 503-506.

Mardia, K.V. \& Jupp, P.E. (2000) Directional Statistics. J. Wiley \& Sons, Chichester.

Mayer, J. (2003) On quality improvement of scientific software: theory, methods, and application in the GeoStoch Development. Doctoral dissertation, University of Ulm.

Mayer, J., Schmidt, V. \& Schweiggert, F. (2004) A unified simulation framework for spatial stochastic models. Simulation Modell. Prac. Theory, 12, 307-326.

Meyer, F. (1989) Skeletons and perceptual graphs. Signal Processing, 16, 335-363.

Omary, M.B., Ku, N.-O., Liao, J. \& Price, D. (1998) Keratin modifications and solubility properties in epithelial cells and in vitro. Subcell. Biochem. 31, 105-140.

Portet, S., Arino, O., Vassy, J. \& Schoevaert, D. (2003) Organization of the cytokeratin network in an epithelial cell. J. Theor. Biol. 223, 313-333.

Ramaekers, F.C. \& Bosman, F.T. (2004) The cytoskeleton and disease. J. Pathol. 204, 351-354.

Ris, H. (1985) The cytoplasmic filament system in critical point dried whole mounts and plastic-embedded sections. J. Cell Biol. 100, 14741487.

Serra, J. (1988) Image Analysis and Mathematical Morphology. Academic Press, London.

Seufferlein, T., Van Lint, J., Liptay, S., Adler, G. \& Schmid, R.M. (1999) Transforming growth factor alpha activates Ha-Ras in human pancreatic cancer cells with Ki-ras mutations. Gastroenterology, 116, 14411452 .

Sewell, J.M., Smyth, J.F. \& Langdon, S.P. (2005) Role of TGF $\alpha$ stimulation of the ERK, PI3 kinase and PLCgamma pathways in ovarian cancer growth and migration. Exp. Cell Res. 304, 305-316.

Soille, P. (2003) Morphological Image Analysis. Springer, Berlin.

Stoyan, D., Kendall, W.S. \& Mecke, J. (1995) Stochastic Geometry and its Applications. J. Wiley \& Sons, Chichester.

Strnad, P., Windoffer, R. \& Leube, R.E. (2002) Induction of rapid and reversible cytokeratin filament network remodeling by inhibition of tyrosine phosphatases. J. Cell Sci. 115, 4133-4148.

Svitkina, T.M. \& Borisy, G.G. (1998) Correlative light and electron microscopy of the cytoskeleton of cultured cells. Meth. Enzymol. 298, 570592.

Vassy, J., Beil, M., Irinopoulou, T. \& Rigaut, J.P. (1996) Quantitative image analysis of cytokeratin filament distribution during fetal rat liver development. Hepatology, 23, 630-638. 
Vassy, J., Portet, S., Beil, M., Millot, G., Fauvel-Lafève, F., Karniguian, A., Gasset, G., Irinopoulou, T., Calvo, F., Rigaut, J.P. \& Schoevaert, D. (2001) The effect of microgravity on cytoskeleton architecture and proliferation of human breast cancer cell line MCF-7. FASEB J. 15, 1104-1106.

Verkhovsky, A.B., Chaga, O.Y., Schaub, S., Svitkina, T.M., Meister, J.J. \& Borisy, G.G. (2003) Orientational order of the lamellipodial actin network as demonstrated in living motile cells. Mol. Biol. Cell, 14, 4667-4675.

Vincent, L. \& Soille, P. (1991) Watersheds in digital spaces: an effcient algorithm based on immersion simulations. IEEE PAMI, 13, 583-598.

Wagner, M., Greten, F.R., Weber, C.K., Koschnick, S., Mattfeldt, T.,
Deppert, W., Kern, H., Adler, G. \& Schmid, R.M. (2001) A murine tumor progression model for pancreatic cancer recapitulating the genetic alterations of the human disease. Genes Dev. 15, 286-293.

Wehrle-Haller, B. \& Imhof, B.A. (2003) Actin, microtubules and focal adhesion dynamics during cell migration. Int. J. Biochem. Cell Biol. 35 , 39-50.

Windoffer, R., Woll, S., Strnad, P. \& Leube, R.E. (2004) Identification of novel principles of keratin filament network turnover in living cells. Mol. Biol. Cell, 15, 2436-2448.

Zackroff, R.V. \& Goldman, R.D. (1979) In vitro assembly of intermediate filaments from baby hamster kidney (BHK-21) cells. Proc. Natl Acad. Sci. USA, 76, 6226-6230. 